\title{
Corela
}

Cognition, représentation, langage

HS-35 | 2022

Anaphore et pronoms en anglais : convergences, différences et complémentarité de quelques approches linguistiques

\section{Anaphore et pronoms en anglais : convergences, différences et complémentarité de quelques approches linguistiques}

\section{Laure Gardelle et Laurence Vincent-Durroux}

\section{OpenEdition}

\section{Journals}

Édition électronique

URL : https://journals.openedition.org/corela/14439

DOI : 10.4000/corela.14439

ISSN : 1638-573X

Éditeur

Cercle linguistique du Centre et de l'Ouest - CerLICO

\section{Référence électronique}

Laure Gardelle et Laurence Vincent-Durroux, « Anaphore et pronoms en anglais : convergences, différences et complémentarité de quelques approches linguistiques », Corela [En ligne], HS-35 | 2022, mis en ligne le 12 janvier 2022, consulté le 24 janvier 2022. URL : http://journals.openedition.org/ corela/14439; DOI : https://doi.org/10.4000/corela.14439

Ce document a été généré automatiquement le 24 janvier 2022.

\section{cc) (†) ()}

Corela - cognition, représentation, langage est mis à disposition selon les termes de la licence Creative Commons Attribution - Pas d'Utilisation Commerciale - Partage dans les Mêmes Conditions 4.0 International. 


\title{
Anaphore et pronoms en anglais : convergences, différences et complémentarité de quelques approches linguistiques
}

\author{
Laure Gardelle et Laurence Vincent-Durroux
}

1 Cet article de présentation concerne deux notions, l'anaphore et les pronoms, dont nous examinons d'abord successivement l'état actuel des connaissances et des problématiques afférentes. Pour chaque notion, nous présentons aussi des éléments complémentaires issus de l'examen d'un corpus commun en anglais par des chercheurs qui évoluent dans des approches différentes, éléments que nous mettons en dialogue. La dernière partie de l'article vise à mettre en évidence la contribution que peut apporter ce nouveau regard croisé pour les deux notions prises ensemble. Nous avons choisi de traiter ces deux notions dans le même volume car elles entretiennent des relations complexes : l'anaphore est souvent illustrée et décrite par les pronoms mais tous les pronoms ne sont pas anaphoriques, et lorsqu'ils le sont, ils véhiculent aussi parfois d'autres valeurs. Cet article constitue donc une introduction à la lecture détaillée des différentes contributions du volume ${ }^{1}$.

\section{L'anaphore}

2 Si l'anaphore s'impose comme phénomène pouvant gagner à engager le dialogue entre chercheurs de diverses approches, c'est parce que les études ont été longtemps scindées entre générativistes et autres chercheurs, les premiers s'intéressant avant tout aux contraintes grammaticales au sein d'une proposition, les autres aux choix discursifs à plus grande échelle. Or il s'est peu à peu avéré qu'un seul modèle ne pouvait tout expliquer, comme va le montrer en résumé le panorama suivant; des ponts ont commencé à s'établir, mais restent limités à ce jour. 


\subsection{L'anaphore dans le domaine nominal : une délimitation en débat}

3 C'est dans le domaine nominal que les recherches sont les plus nombreuses et les plus complexes. Il serait trop long ici de retracer toutes les études; l'objectif de cette section est plutôt de faire apparaître les grands positionnements théoriques et leurs points de divergence ou convergence, en faisant le moins d'injustice possible à la profusion des recherches, malgré d'inévitables simplifications.

4 Les générativistes, par le biais de la Théorie du Liage, se sont surtout intéressés aux contraintes grammaticales au sein d'une proposition donnée. Le point d'entrée a été l'anaphore coréférentielle (et cas proches de variable liée ou de pronoms de type E) : il s'est agi d'expliquer les contraintes interprétatives différentes qui pèsent sur le pronom personnel réfléchi, le pronom personnel (simple) et le syntagme nominal. Par exemple, Bill looked at himself impose la coréférence entre himself et Bill, alors que him ou Bill substitués à himself l'excluent. La notion de "commande » initialement introduite par Langacker (1969) est optimisée par celle de «c-commande » de Reinhart (1976, 1981) - en anglais, c-command pour constituent-command. ${ }^{2}$ La Théorie du Liage définit ainsi trois types d'expressions :

5 - les « anaphoriques " ('anaphors', entendus dans le sens restreint de pronoms liés : réfléchis, réciproques liés, ex. himself), qui doivent être liés par leur antécédent dans leur domaine local ;

6 - les « pronoms » ('pronominals', c'est-à-dire les pronoms, dont les possessifs, non liés, ex. him), qui doivent être libres dans leur domaine local, mais peuvent être liés hors de leur domaine local ;

7 - les « expressions $\mathrm{R}$ » ('R[eferential]-expressions', c'est-à-dire des groupes nominaux, ex. Bill), qui doivent être libres dans tous les domaines (pour plus de détails, voir par exemple Haegeman \& Guéron 1999 : 362-383 ou Sportiche et al. 2014 : 157-186).

8 La Théorie du Liage conçoit donc l'anaphore comme un phénomène syntaxique contraignant : les trois types d'expressions sont mutuellement exclusifs. Elle ne nie pas l'existence de relations anaphoriques à une échelle plus grande du discours, mais il ne s'agit plus de syntaxe ; ces relations sont donc hors du champ d'étude. ${ }^{3}$ Reinhart les nomme cas d'« anaphore libre » (Zribi-Hertz 1996 : 92).

9 Or ce sont précisément ces relations libres qu'étudient d'autres champs de la linguistique: sémantique, linguistique cognitive, linguistique énonciative, pragmatique, psycholinguistique notamment. Elles font apparaître un certain nombre de problèmes que l'approche générativiste ne peut résoudre, et surtout une très grande complexité du processus anaphorique, qui n'est pas totalement modélisée à ce jour.

10 Tout d'abord, si l'anaphore coréférentielle a longtemps retenu l'attention, un certain nombre de recherches ont montré l'occurrence fréquente d'anaphores dites indirectes (connues en anglais sous le nom de bridging anaphora, voir par exemple Irmer 2011), c'est-à-dire qui nécessitent des inférences pour l'interprétation de l'anaphorique :

11 - les plus connues sont les anaphores associatives, toujours avec un article défini pour l'anglais ou le français. On parle d'«association" car bien que l'antécédent et le segment anaphorique ne soient pas coréférentiels, le référent de l'anaphorique est activé "par association" par la mention du référent de l'antécédent. ${ }^{4}$ Ainsi : Grignon demande alors à Chapelain de lui servir de guide jusqu'au village voisin de La Flocelière. $\underline{\text { Le }}$ 


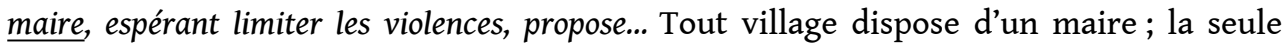
mention d'un village suffit donc à rendre actif cet autre référent.

12 mène strictement textuel, mais synonyme plutôt de préconstruction; en effet, étymologiquement, "anaphore " signifie simplement "porter en arrière", donc reprendre du déjà posé. C'est ce que propose la linguistique énonciative, dans les analyses de THE en particulier, toujours anaphorique parce qu'il implique la préconstruction, le «déjà pensé ». Ainsi Lapaire \& Rotgé $(1991: 118,122)$ qualifient d'« anaphore culturelle » la référence à ce qui est acquis culturellement (ex. the moon), ou encore d'" anaphores situationnelles" des cas tels que See, down there, among the bushes; l'anaphore se distingue de la deixis par le fait que l'existence du référent est considérée comme acquise au moment de l'énonciation, plutôt que nouvelle. Ou encore, Cotte (1996: 198) nomme "anaphore intérieure " un cas tel que the fiction that they write : le syntagme étant issu de they write fiction, «la notion fiction est repérée dans la proposition primitive dont elle a été extraite, elle en a intégré le procès et en est définie ». En d'autres termes, la préconstruction et la reprise se situent dans la genèse du syntagme plutôt que dans la linéarité du discours. Mignot (2016:113) propose de nommer ces cas sans première mention « anaphore de construction", à distinguer de l'« anaphore textuelle».

17 La conception de l'anaphore comme du préconstruit en genèse conduit un certain nombre d'énonciativistes à considérer également comme anaphoriques d'autres opérateurs, dont certains n'ont pas vocation à référer: tout terme porteur du morphème TH-, marqueur d'« anaphore opérationnelle » pour Lapaire \& Rotgé (1991 : 238), ainsi THEN ou THERE ; mais aussi, par exemple, So (par opposition à AS) ou -ING, « outil d'anaphorisation » (ex. Lapaire \& Rotgé $1991: 417){ }^{5}$ 
de la limite entre anaphore et deixis; ainsi Allan (2009) cite comme cas d'anaphore l'exemple (2) suivant, traditionnellement posé comme deixis :

(2) (cité par Allan 2009: 464) (en reprenant son manteau des mains de l'employé d'un vestiaire) This is torn!

19 Ou encore, Cornish (1999), à la suite d'Ehlich (1982), considère que les anaphoriques sont seulement les SN définis et les pronoms personnels, tandis que les démonstratifs sont toujours anadéictiques (pour un panorama plus détaillé, voir également Gardelle et al. 2019).

Les approches cognitives de la référence vont jusqu'à poser la question de l'utilité même du concept d'anaphore. L'Echelle des marqueurs d'accessibilité d'Ariel (ex. 1990, Accessibility Marking Scale), la Hiérarchie du Donné de Gundel et al. (1993, Givenness Hierarchy) proposent des degrés d'accessibilité ou de donné pour des expressions référentielles, indépendamment de leur statut anaphorique ou non. Par exemple, Ariel propose l'échelle reproduite en (3): les formes linguistiques correspondent intrinsèquement à des degrés plus ou moins élevés d'accessibilité du référent, et signalent ceci. Plus on va à droite de l'échelle, plus les marqueurs signalent un faible degré d'accessibilité de l'élément par rapport aux précédents.

(3) Echelle de marqueurs d'accessibilité d'Ariel (1990:33):

zéro < réfléchis < marqueurs d'accord < pronoms clitiques < pronoms inaccentués < pronoms accentués < pronoms accentués + geste < démonstratif proximal $(+\mathrm{SN})<$ démonstratif distal $(+\mathrm{SN})<$ démonstratif proximal $(+\mathrm{SN})+$ modifieur $<$ démonstratif distal (+ SN) + modifieur < prénom < nom de famille < description définie courte < description définie longue < prénom et nom $<$ prénom et nom + modifieur

Parallèlement, sous l'impulsion notamment de Schnedecker (ex. 1997, 2014), le concept d'anaphore est partiellement concurrencé dans des études récentes par celui de " chaîne de référence », créé par Chastain (1975), parce qu'il y est jugé plus opératoire pour étudier certains phénomènes interprétatifs. Une chaîne de référence se définit comme « la suite des expressions d'un texte entre lesquelles l'interprétation construit une relation d'identité référentielle " (Corblin 1995: 23). Longue d'au moins trois maillons, elle permet de dépasser la relation à deux membres qu'isole l'anaphore (Schnedecker \& Landragin $2014: 4$ ) ; elle prend également en compte le fait que même si l'on répète un nom propre, par exemple, bien que l'accès à la référence ne nécessite pas de segment antécédent, une seconde mention intègre les connaissances apportées depuis la première mention, un point commun non négligeable avec les cas qualifiés d'anaphores.

En effet, comme l'ont montré notamment Reichler-Béguelin (1993) puis Cornish (1999), dans le processus anaphorique, le segment "antécédent» n'est en fait qu'un «introducteur textuel», un «déclencheur d'antécédent» ('antecedent trigger') respectivement : il a seulement pour rôle d'introduire le référent en discours. Pour Cornish, il serait plus juste de nommer "antécédent " la représentation mentale de l'entité de discours ('mentally represented discourse entity') :

(4) (Cornish 1999: 44) The antecedent trigger introduces an entity into the discourse via its predicational and utterance context, and an anaphor of a particular type and form accesses that mentally represented discourse entity at a later point in the discourse, adding to this representation further properties resulting from the processing of the anaphoric clause as a whole. last night, but it was much too acid for his liking: 
24 - l'antécédent an apple introduit le référent dans le discours, dans le contexte de la proposition qui le contient ; l'image mentale est celle de la pomme mangée, ou en train d'être mangée, par Joe ;

25 - puis it donne accès au référent dans la représentation mentale à un stade ultérieur du discours. Cette représentation intègre les informations données en amont, mais aussi celles qui sont apportées par le reste de la proposition. Parler du goût (acid) véhicule une représentation de la pomme en train d'être mangée, ou terminée.

26 Enfin, les études en psycholinguistique (acquisition L1, acquisition L2, interaction, publics hors de la norme) ont montré que loin d'un procédé mécanique encodé par le locuteur, l'anaphore était, en contexte de communication, un procédé de coconstruction (Gardelle et al. 2019 ; David et al. 2019 ; Debras \& Beaupoil-Hourdel 2019). Locuteur et allocutaire s'adaptent perpétuellement l'un à l'autre; un locuteur peut également marquer sa domination par des choix propres de catégorisation. Par exemple, dans un entretien clinique étudié par Salazar-Orvig \& Grossen (2010) (énoncé 5 ci-dessous), le psychiatre ne se contente pas de marquer sa compréhension du référent lorsqu'il utilise l'anaphorique ces rêves-là en fin d'extrait. Catégoriser les scènes décrites comme des rêves (plutôt que cauchemars employé plus haut par la patiente) marque une prise de distance par rapport aux propos de la patiente, et une tentative d'imposer une nouvelle représentation.

(5) (Salazar-Orvig \& Grossen 2010: 7) Locuteurs : Psychiatre (PSY), Lamia (LAM)

patiente

PSY 143 - non? vous ne rêvez pas?

LAM 110- $j(e)$ suis comme $j(e)$ suis nerveuse $j(e)$ fais plutôt des cauchemars.

PSY 144 - ah ah comment c'est les cauchemars?

LAM 111 - ben je rêve que mon père il me tue.

PSY 145 - vous pouvez nous raconter plus précisément un rêve?

LAM 112 - ouais je rêve que mon père il m'enlève, qu'il m'enlève de la France, qu'il

m'emmène en Algérie +\# qu'il me marie de force euh, que j'ai un mari qui me bat, ++

que j'ai des enfants +++

PSY 146 - mhm

LAM - ma belle-mère elle me fouette, + des choses comme ça, je vois des serpents

des- partout des araignées, des trucs comme ça.

PSY 147 - et depuis depuis longtemps vous faites ces rêves-là ?

\subsection{L'anaphore verbale : un point moins étudié}

27 L'anaphore verbale a également fait l'objet d'études à la fois en syntaxe générative et en linguistique énonciative, principalement par le biais des formes en DO X : do it / this / that / so, auxquels peuvent être ajoutés do the same thing, do otherwise, do something else, do likewise (Culicover 2009: 454). Il s'agit d'un point beaucoup moins étudié que l'anaphore nominale, et il n'est pas recensé comme "anaphore » dans les deux ouvrages de référence d'introduction à l'analyse syntaxique générative consultés (Haegeman \& Guéron 1999, Sportiche et al. 2014).

En syntaxe générative, la majeure partie des recherches vise à comprendre la distinction entre DO X et d'autres formes de syntagmes verbaux : DO seul, ellipse postauxiliaire (également considérés comme anaphoriques par Miller 2013: 123 notamment). A ce titre, Culicover \& Jackendoff (2005: 284) et Culicover (2009: 454) considèrent do so comme "représentatif » des autres, par opposition à l'ellipse postauxiliaire; ils peuvent ainsi résumer : «Do $X$ anaphora, of which we take do so as 
representative, requires its antecedent to be an action » (Culicover \& Jackendoff 2005 : 284). Parmi les linguistes d'inspiration au moins en partie générativiste, seuls Miller (ex. 2011, 2013, ou encore l'article de ce volume) et Flambard (2017), à notre connaissance, étudient les différences de contraintes au sein des formes en DO X, et optent pour une approche compositionnelle, considérant l'apport du pronom. En cela, ils établissent un pont avec les approches énonciativistes, qui, elles, partent de la contribution de chacun des termes pour rendre compte au moins partiellement des usages.

La démarche énonciativiste, qui se concentre sur les différences entre les formes de DO X, qualifiées de "proformes complexes» (ex. Lapaire \& Rotgé 1991: 521), est bien illustrée par l'extrait suivant :

(6) (Lapaire \& Rotgé 1991 : 522) DO est, dans cet emploi, très étroitement associé à un substitut anaphorique patenté - THIS, THAT, IT, SO - avec lequel il « reprend » un verbe et ses compléments, présents dans le contexte-avant. [...] [I]l ne faut pas perdre de vue que sans la présence de THIS, THAT, IT ou SO le processus de substitution serait gravement compromis. Ceci laisse entendre que le pouvoir de «remplacement» des proformes complexes DO THIS / THAT / IT / SO est plus l'œuvre de THIS / THAT / IT / SO que celle de DO. sont d'ailleurs traitées dans le chapitre sur IT. Les énonciativistes avancent l'idée d'un invariant pour chacun de ces opérateurs, dont la contribution explique au moins en partie les contraintes ou préférences constatées. Pour Lapaire \& Rotgé (1991 : 223), « IT signale une reprise très décolorée, qui est au-delà du débat entre clôture anaphorique (THAT) et non-clôture (THIS), et qui ne transmet aucune information marquée sur la manière, la congruence ou la validation (SO). » Ou encore, Gardelle \& Lacassain-Lagoin (2012: 299) proposent de voir avec SO une perception du procès comme une manière de faire - une manière conventionnelle d'agir, par exemple.

Flambard (2017), à la suite de différentes grammaires, explique les différences entre DO So et les autres par le paramètre de l'agentivité, préférée (ou parfois exigée) pour les autres DO X (\$49) mais pas pour DO SO, qui admet même des antécédents statiques (\$64), ainsi dans l'extrait suivant, emprunté à Houser (2010:53): The three clusters with fewer matches seem to exhibit a mixture of expression patterns, while the first cluster does so to a much lower extent. Quant à la distinction entre démonstratifs et IT, elle repose sur le statut informationnel du procès évoqué : IT suppose souvent un référent bien connu, là où les démonstratifs impliquent une forme de nouveauté. Là, l'auteur reprend implicitement la conception énonciativiste de l'invariant: THIS indique souvent une forme de proximité avec le locuteur, THAT une forme de distance (\$72). Un pont est également implicitement établi avec les théories cognitives de la référence : un référent désigné par IT est considéré comme plus accessible à l'interlocuteur qu'un référent désigné par un démonstratif (Ariel 1990, Gundel et al. 1993). Miller (2011) y fait explicitement référence :

(7) (Miller 2011: 9) As for the contrast between do it and do this and do that, a detailed study is beyond the scope of this paper. However, it is clear that the gricean quantity effects described by Gundel et al. [4] do apply in these cases. Use of do that or do this in contexts where do it is sufficient to recover the intended antecedent leads the addressee to attempt to recover a less accessible antecedent with infelicitous results. » 


\subsection{Quel dialogue, quels apports sur l'anaphore, dans ce volume?} publiées dans ce volume sont les suivants : comment prendre en compte à la fois les cas plus contraints (intra-propositionnels) et ceux, plus tributaires d'enjeux pragmatiques, à une échelle de discours plus large ? Quelle est la place du sens ? Comment décrire le lien entre anaphorique et antécédent, et donc quelles limites d'application retenir pour l'anaphore ? De plus, à quoi le concept sert-il d'un point de vue métalinguistique - est-il même utile? Enfin, quelle est la meilleure méthodologie d'étude : les cas d'école cités dans les grammaires, qui font apparaitre un segment antécédent nominal et un anaphorique nominal ou pronominal, sont-ils à faire passer au second plan ? C'est à ces questions que les différents auteurs ont finalement contribué à répondre au travers de leurs analyses du corpus commun. Voici quelques lignes saillantes qui ressortent des échanges suscités par le croisement des différentes approches.

Parmi les points de convergence forts figure la définition même de l'anaphore. Tous retiennent l'idée d'un segment dont l'interprétation dépend d'autre chose introduit antérieurement ; il est apparu en outre utile de distinguer l'« anaphore » de la simple " préconstruction ». Pour -ING ou the end of the film, par exemple, il est apparu au terme des échanges que le terme d'anaphore n'était finalement pas indispensable, pouvant conduire à des amalgames. Le point commun est une forme de cumul d'informations, en plusieurs étapes, mais là, il n'a pas vraiment lieu dans la linéarité du discours. Il est également apparu que l'« anaphore » était à distinguer du " défini », avec lequel elle est seulement en lien; Bill, ou people's lives, sont définis, mais pas anaphoriques. La cataphore a également fait l'objet de mises au point largement partagées : mieux vaut parler d'« anaphore anticipatoire" (en anglais, backwards / anticipatory anaphora) lorsque la syntaxe vise seulement à une mise en attente de l'antécédent (ex. Before I noticed him, a thief...).

Un deuxième point de convergence fort est la distinction irréductible à établir entre deux niveaux d'étude : un niveau intra-propositionnel, dans lequel les contraintes sur l'interprétation sont plus fortes, comme si l'anaphore était en quelque sorte "grammaticalisée ", et un niveau plus libre à une échelle plus grande. Il apparaît impossible de modéliser précisément le procédé sans cette distinction : au niveau intrapropositionnel, il ne peut exister que des compatibilités entre l'apport opérationnel des formes et les contraintes qui pèsent sur leur référence, plutôt que des motivations vivantes en discours. A l'inverse, anaphores indirectes ou associatives, implicatures conversationnelles, co-construction, choix énonciatifs, effets intonatifs, ne concernent que l'échelle inter-propositionnelle. Il s'agit là d'une richesse qui découle précisément de l'existence moindre de contraintes fortes. Dans le domaine verbal, ces deux niveaux ne sont pas pertinents, puisque l'antécédent ne peut se trouver dans la même proposition que l'anaphorique; mais il faut distinguer malgré tout les cas syntaxiquement ou sémantiquement contraints (ex. compléments nuls, ainsi even though he tried $\varnothing$; ou encore, pour l'anaphore en DO X, seul DO SO admet communément un référent non agentif) et ceux qui relèvent d'un choix énonciatif. DO SO diffère alors plus que les trois autres (DO IT / THIS / THAT); de même, lui seul apparaît comme une proforme complexe, au sens où il ne se subdivise pas en verbe + objet. En résumé, il est apparu qu'une analyse en "tout sémantique » ne pouvait rendre compte de toutes les contraintes constatées; le sémantique (ou la contribution procédurale d'un opérateur) 
motive parfois les usages, mais n'est parfois que compatible avec la contrainte notée, plutôt qu'à l'origine de la contrainte.

L'étude d'un corpus oral, une conférence visant à faire adhérer un public, a par ailleurs conduit à mettre en évidence le rôle majeur du co-énonciateur, plus que ne le font habituellement la majorité des approches représentées : il ne s'agit pas juste, voire pas toujours, de s'adapter au co-énonciateur pour se faire comprendre de lui, mais aussi de trouver, par la préconstruction, une forme d'accord avec lui, voire d'imposer volontairement un positionnement (comme lorsque Tony Robbins se pose en expert via I get the phone call). Le co-énonciateur doit lui aussi s'adapter, le référent n'étant pas toujours clairement identifiable.

On en vient à l'utilité même du concept d'anaphore. Comme l'a indiqué Pierre Cotte durant un échange, si le concept est apparu initialement en linguistique, c'est pour rendre compte des pronoms personnels de $3^{\mathrm{e}}$ personne - d'abord analysés comme des "substituts abréviatifs", pauvres au regard d'un syntagme nominal complet, mais reconnus aujourd'hui comme riches, porteurs d'un statut cognitif propre : signaler une continuité d'attention. Or un certain nombre de phénomènes notés à propos de l'anaphore ne lui sont pas propres. Par exemple, bien que je ne soit pas anaphorique, un texte qui regorge d'occurrences de je pour un même locuteur invite à agréger les informations énoncées à propos du référent, comme dans le processus anaphorique. Ou encore, de même que certains anaphoriques sont plus riches lexicalement que d'autres (ex. the doctor plutôt que he), il existe hors anaphore des descriptions définies plus longues que d'autres; de même, lexicalement, les hyperonymes constituent des formes moins riches que leurs hyponymes. Les divers auteurs concluent à la nécessité de prendre en compte ces autres phénomènes pour cerner les spécificités de l'anaphore, ce qui n'est pas suffisamment fait aujourd'hui dans les recherches. Cela ne signifie pas que le concept d'anaphore n'est pas pertinent; bien au contraire. Par exemple, les chaînes de référence, considérant trois maillons au minimum, ne peuvent rendre compte de certaines contraintes notées en particulier par la Théorie du Liage. Une attention plus grande devrait également être portée à la dimension phonologique. Les pronoms accentués sont au mieux brièvement évoqués dans les études existantes, or $\mathrm{S}$. Wilhelm (ce volume) prône un statut linguistique des éléments suprasegmentaux pour marquer l'Accessibilité d'un référent.

Quant à la méthodologie, s'il est important de recourir à des occurrences qui se trouvent aux limites du phénomène, tous concluent qu'il est tout aussi essentiel de prendre en compte des cas "typiques", d'acceptabilité sûre. De plus, les jugements individuels sur des énoncés manipulés sont parfois difficiles à donner; les énoncés authentiques, disponibles en grand nombre grâce aux corpus électroniques notamment, apparaissent comme la meilleure base d'analyse.

Ces convergences ne doivent pas faire oublier les différences entre les approches, dont la lecture détaillée des articles permet de prendre la pleine mesure. L'idée d'un invariant, ou du moins d'une continuité des valeurs d'un opérateur donné, n'est pas une préoccupation des études générativistes ou partiellement générativistes (ainsi $\mathrm{P}$. Miller, ce volume) ; elle est au contraire fondamentale pour les énonciativistes. Les cas d'emploi syntaxiquement contraint sont au contraire peu étudiés par ces approches. L'approche pragmatique (J.Albrespit dans ce volume) place au premier plan les négociations entre locuteur et allocutaire, là où l'autre n'est guère important pour les générativistes. Ce qui ressort peut-être principalement pour le lecteur extérieur, c'est 
l'utilité de chacune de ces approches pour rendre saillant tel ou tel paramètre ; c'est aussi la nécessité d'un certain dosage de ces paramètres, certains apparaissant plus ou moins influents selon le contexte de communication ou la configuration syntaxique. C'est là que le dialogue s'avère indispensable si l'on veut parvenir peu à peu à une modélisation fine et complète de ce processus particulièrement complexe qu'est l'anaphore.

\section{Les pronoms}

On l'a vu, les pronoms, notamment les pronoms personnels (et en partie les démonstratifs), sont historiquement indispensables à l'existence même du concept d'anaphore; aujourd'hui encore, ils sont largement représentés dans les études sur l'anaphore, sans doute parce qu'ils permettent de mettre au jour les caractéristiques principales $\mathrm{du}$ procédé: saillance cognitive, amplitude variable du référent, fonctionnement syntaxique etc. Mais le recoupement n'est que partiel: comme l'a rappelé Jean-Marie Merle dans un échange, «pronom» nomme une catégorie syntaxique, tandis que l'« anaphore» désigne un rôle. Les pronoms n'ont pas tous vocation à avoir un rôle anaphorique (certains sont déictiques, d'autres sont non référentiels) et les frontières sont parfois floues en discours entre interprétation anaphorique et interprétation déictique, référence endophorique et référence exophorique.

La catégorie des pronoms, déjà du fait de son appellation, entretient également des liens avec celle des noms; nous envisageons ci-dessous quelques-uns de ces liens, qui se révèlent complexes. Par ailleurs, alors que les pronoms se présentent habituellement sous une forme écrite et orale très brève, et constituent une catégorie restreinte, ils sont aptes à intégrer une composante sémantique souvent plurielle; nous en évoquons ensuite les principales configurations.

\subsection{Les liens complexes entre pronoms et noms}

41 Les intitulés attribués aux deux catégories, "pronoms » et " noms ", sont très proches une lecture immédiate peut favoriser une interprétation simplificatrice selon laquelle les termes d'une catégorie (celle des pronoms) seraient destinés à se substituer aux termes de l'autre (celle des noms), et ce, grâce à des propriétés communes. La notion de substitution entre un nom et un pronom se heurte cependant rapidement à ses propres limites, puisque la plupart des pronoms sont en fait coréférents à un groupe nominal plutôt qu'au seul nom, mais aussi parce qu'ils peuvent être coréférents à des tournures verbales, voire prédicatives comme dans l'exemple suivant: I wrote three letters to my sister. I did it in order to... De plus, la conception traditionnelle du pronom qui se substitue au nom est mise à mal par Blanche-Benveniste et al. (1987) qui proposent que les pronoms soient en fait les formes de base (voir plus bas) plutôt que les formes secondes. Pronoms et noms pourraient même constituer une seule catégorie, selon le point de vue adopté.

\subsubsection{Des morphèmes et des propriétés partagés}

Sans surprise, les deux catégories ne sont pas étanches : elles partagent des morphèmes mais aussi des propriétés. Ainsi, on constate par exemple que des termes nominaux tels 
que body ou thing, associés aux quantifieurs any, some ou no, apparaissent dans la catégorie pronominale: somebody, something, anybody, anything, nothing... L'origine nominale de ces termes reste bien visible à l'écrit mais elle n'est que faiblement perçue à l'oral: l'inaccentuation et la voyelle réduite associée indiquent l'affaiblissement sémantique du terme d'origine nominale. Les termes nominaux constitutifs sont parfois qualifiés de classificateurs (Adamczewski, 1982:261) pour leur aptitude à créer une classe sur la base de traits sémiques minimaux. L'existence de cette classe se révèle dans le choix du pronom de reprise, avec par exemple la reprise de someone ou somebody par they (voir Gardelle \& Lacassain-Lagoin 2012 : 70-71) : pour le pronom personnel en contexte générique, l'« accord " sémantique semble prévaloir sur l'accord syntaxique, soulignant alors la représentation prépondérante de la multiplicité des référents potentiels, de leur indétermination et encore une fois de la coréférence.

D'autre part, certains pronoms peuvent, dans une certaine limite, se comporter comme des noms en acceptant une forme de détermination : par exemple, where peut porter un quantifieur (anywhere, somewhere, nowhere). Il en est de même pour le pronom one (anyone could say that).

Une autre propriété commune est que le genre et le nombre affectent la catégorie nominale comme la catégorie pronominale, avec une capacité étendue du pronom à indiquer le genre : si les noms en anglais n'ont pas de marque flexionnelle de genre et laissent parfois transparaître le genre naturel (cat ou child ne spécifient pas le genre, contrairement à son ou daughter), he, she et it apportent systématiquement une information de genre (voir notamment Gardelle 2006, Mignot 2012). Ces informations de genre, en anglais, ne concernent cependant pas tous les pronoms ; par exemple, au sein des pronoms personnels, il s'agit seulement de la $3^{\mathrm{e}}$ personne du singulier. Quant au nombre, il est porté par les noms, grâce à la détermination, comme par certains pronoms, avec des formes spécifiques de singulier et de pluriel, telles que me / us; en revanche, certains pronoms n'indiquent pas le nombre, tels que les possessifs (mine, yours...).

45 Enfin, dans ce rapide panorama, il convient de mentionner que les deux catégories accèdent aux mêmes fonctions syntaxiques, celles de sujet et de complément du verbe.

\subsubsection{Des catégories spécifiques}

Toutefois, les rapprochements évoqués ne doivent pas masquer la spécificité de chaque catégorie, ni simplifier leur rôle l'une par rapport à l'autre.

Ainsi, Creissels (1993 : 63) remet en cause la définition courante du pronom comme " mis pour un nom ", jugée imprécise. Creissels relève qu'on peut donner à la " notion » de pronom un «contenu discursif, en identifiant comme pronom toute unité qui reprend le référent d'un constituant nominal présent dans le contexte, ou bien un contenu syntaxique en limitant le terme de pronom à des unités qui occupent dans la phrase une position de constituant nominal mais dont la structure interne n'est pas celle d'un syntagme nominal constitué d'un noyau substantival éventuellement assorti de diverses déterminations ». Illustrant cette distinction par des «pronoms » du français, Creissels (ibid.) indique que, selon la première interprétation, le statut de pronom ne peut pas être reconnu à « moi », qui ne reprend pas un constituant nominal présent dans le contexte et n'a donc pas de "contenu discursif», alors que selon la 
deuxième interprétation, «le » dans « je le connais » ne peut pas être reconnu comme pronom car, placé devant le verbe, il n'occupe pas une position de constituant nominal.

En prolongement de cette prise de position, il y a lieu de mentionner l'approche dite « pronominale ». Cette approche, inspirée de Quine (1964:17) ("Pronouns are the basic media of reference; nouns might better have been named propronouns") est reprise par Stefanini (1976 : 317) et explicitée ainsi : « loin d'être à la place du nom, le pronom, nous pourrions le concevoir, est antérieur au nom. Il existe des démonstratifs comme ceci qui signifiera un objet même pour qui on ignore le nom. Beaucoup de choses n'ont pas de nom: on les conçoit à l'aide d'un pronom démonstratif ». En d'autres termes, selon Blanche-Benveniste et al. (1987), l'approche pronominale pose que dans la relation entre pronoms et formes lexicales de noms, ce sont les pronoms qui sont les formes de base, en nombre restreint, à partir desquels s'effectuent les processus de lexicalisation. Ces auteurs distinguent ainsi une sémantique "primitive» et une sémantique " seconde ", la première correspondant à un noyau verbal et permettant de constituer un groupe restreint de phrases pronominales pour l'analyse. Le pronom est premier parce qu'il constitue la «base linguistique de l'énoncé»: il marque la "sémantique primitive", au sens où il préfigure la fonction syntaxique qui sera accordée aux items lexicaux (Blanche-Benveniste et al. 1987:27). Ainsi, dans une proposition, c'est par le biais des pronoms, notamment interrogatifs, que les relations argumentales organisées par le verbe peuvent être mises au jour; donner invite par exemple à se poser ces questions: "qui a donné quoi, à qui, comment, où, etc.» (Buyssens 1967 : 110). Le lexique, quant à lui, ne constituerait que la «sémantique seconde » : il serait ajouté par un procédé de lexicalisation.

L'approche pronominale, même si elle s'est assez peu propagée dans les études linguistiques, présente certains avantages. D'une part, elle explique pourquoi le pronom permet de définir l'unité-syntagme : quand un pronom est impossible, c'est qu'il n'y a pas de SN (ex. Il a raison: avoir raison est un verbe complexe et non une séquence verbe $+\mathrm{SN}$ objet car ${ }^{*} I l$ a quoi? est impossible) (ibid.). D'autre part, le caractère primitif du pronom personnel permet d'expliquer pourquoi en anglais, les énoncés météorologiques (ex. let it rain), ou encore un certain nombre de locutions dans lesquelles le pronom n'a aucun référent identifiable (ex. go it alone, 'se lancer seul', littéralement 'aller-[pronom personnel COD neutre]-seul'), admettent pour certains locuteurs une variation de genre sur le pronom (let her rain / go her alone), mais excluent toute alternance avec un SN développé ( ${ }^{*}$ go the project alone) (Gardelle $\left.2014: 82\right)$. Cette approche permet enfin de ne pas considérer comme exceptionnels les pronoms sans référent, puisque l'affectation de traits sémantiques relève de la sémantique seconde. Elle permet également de rendre compte du fait que les pronoms ne sont pas seulement coréférents à des noms mais également à d'autres catégories : des pronoms peuvent référer en effet à des formes exprimées par des éléments non nominaux, voire non exprimées (cf. ci-dessus, notamment Cornish 1999: 44). En tout état de cause, les pronoms ne résultent pas d'un appauvrissement, bien au contraire, comme en témoigne leur rang élevé dans les hiérarchies d'accessibilité (pour plus de détails, voir la contribution de L. Gardelle dans ce volume).

\subsection{Classification et signification des pronoms}

Si nous nous intéressons maintenant à la catégorie des pronoms pour elle-même, et plus particulièrement à la signification des pronoms, se pose alors inévitablement la 
question des raisons de l'affectation à cette catégorie des éléments qui la constituent. Un nouveau regard, enrichi par des travaux récents, mérite d'être porté sur ces raisons, qui ne sont peut-être pas irréprochables. Notamment, il est nécessaire de tenir compte du fait que les catégories grammaticales ont été définies en observant les langues écrites, fondées sur la représentation de mots isolés graphiquement. Or, la première réalité des langues est leur forme orale, caractérisée par un flux, et il s'avère indispensable d'observer les pronoms tels qu'ils se présentent à l'oral. Selon BlancheBenveniste (2008 : 200-201), les pronoms personnels clitiques du français ne sont traités « comme des mots de plein statut que dans l'écriture » alors qu'ils se comportent en fait à l'oral comme des affixes verbaux. Ces termes devraient alors être exclus de la catégorie des pronoms. Blanche-Benveniste les considère comme des «moins que mots » et rapporte que Meillet tirait déjà de ses observations, publiées dans les années 1930, l'idée que prendre l'écrit pour base d'analyse était une erreur. La formalisation en catégories par l'analyse de la langue orale aurait pu ou pourrait donner lieu à des répartitions différentes.

\subsubsection{Une catégorie non homogène}

51 Par ailleurs, du point de vue de leur signification, la catégorie des pronoms n'est pas homogène. Non seulement elle comporte un nombre non négligeable de souscatégories selon le rôle joué par les pronoms dans l'énoncé (pronoms relatifs, interrogatifs, déictiques), mais en outre, certaines de ces catégories posent problème. C'est le cas notamment des pronoms classiquement désignés comme pronoms personnels, dont Bhat (2004) propose d'exclure les pronoms de $3^{\text {ème }}$ personne (Gardelle 2014: 70) ; voici pourquoi.

Bien que le terme de «pronom personnel » soit traditionnellement utilisé parce que les pronoms distinguent le trait de la personne (locuteur, allocutaire et délocuté respectivement pour la première, deuxième et troisième personnes), ceux dits « de $3^{\mathrm{e}}$ personne » diffèrent en grande partie des autres (Bhat 2004:32, 132-149). Eux seuls relèvent de la non-personne (au sens de Benveniste 1966: 256) et peuvent être anaphoriques, tandis que les pronoms des deux premières personnes ne renvoient qu'à des instances de discours; de ce fait, eux seuls ne sont pas des embrayeurs (shifters). Le genre, lorsqu'il existe à la $3^{\text {e }}$ personne, peut donner des indications sur le référent, alors que lorsqu'il est marqué dans les $1^{\text {ère }}$ et $2^{\text {ème }}$ personnes, il permet plutôt des distinctions sociales (et il y est bien plus rare). Enfin, dans un certain nombre de langues, dont les langues romanes et germaniques, le pronom de $3^{\text {éme }}$ personne n'est pas lié morphologiquement aux pronoms des deux autres personnes, mais est issu diachroniquement du démonstratif, voire emprunte des formes au démonstratif. Par exemple, en kharia, langue munda de l'Est de l'Inde, les pronoms de troisième personne sont obtenus par ajout du suffixe kar aux démonstratifs : $u$ ('this') forme u-kar, ho ('that') ho-kar, han ('that remote') han-kar (Bhat $2004: 133$ ).

Bhat (2004:5) conclut que le terme de "pronom personnel» (personal pronoun) doit être réservé aux pronoms de $1^{\text {ère }}$ et $2^{\text {ème }}$ personne, tandis que ceux de $3^{\text {ème }}$ personne doivent être nommés «proformes» (proforms). Parce que le terme de "proforme » ne pourrait s'appliquer uniquement aux pronoms de troisième personne (en anglais, DO, par exemple, connaît des emplois de proforme), il peut paraître préférable de conserver l'étiquette de " pronom personnel ». Mais la mise en saillance de ces différences permet de conclure à la singularité relative de la $3^{\text {ème }}$ personne. D'ailleurs, en anglais, le 
système verbal intègre cette singularité par une marque encore visible au présent simple au singulier ( $\mathrm{V}$-s) et le système pronominal explicite la non-personne dans l'existence d'un pronom vidé du trait humain, IT.

L'absence d'homogénéité au sein de la catégorie des pronoms est également visible dans la sous-catégorie des pronoms relatifs de l'anglais: WHICH, WHO et THAT. Les pronoms relatifs en WH-, eux, peuvent intégrer le trait + / - humain de leur antécédent et peuvent être précédés par des prépositions, comme le sont les groupes nominaux. Sur ces deux aspects, THAT se démarque (*the house in that I live). Or là encore, ces différences soulèvent la question de l'appartenance même de THAT à la catégorie des pronoms. Pour les générativistes, ce statut est refusé : il est qualifié de conjonction et plus spécifiquement de "complémenteur »: alors que le pronom relatif en WH- monte " en position de spécifieur, laissant une trace vide coréférentielle dans la proposition inférieure, qui ne comporte pas de complémenteur (...) THAT ne peut être que complémenteur " (Khalifa 1999 : 198; voir également Sportiche et al. 2014 : 407). D'autres linguistes qualifient THAT de « relateur », reconnaissant par cette appellation sa nature de relatif atypique, assurant une relation lâche avec son antécédent. Pour d'autres approches et plus de détails, on se référera à van der Auwera (1985). De plus, THAT se démarque des pronoms en WH- par le fait que contrairement à eux, il ne peut être interrogatif. Les différences observées, qui rendent THAT quelque peu singulier, peuvent être rattachées à l'origine différente de deux types de marqueurs : TH- signale un acquis (Lapaire \& Rotgé 1991 : 608) alors que WH- signale un manque.

\subsubsection{Une catégorie trop souvent décrite à l'aune des pronoms personnels}

55 Si la plupart des réflexions rassemblées ci-dessus s'appuient plus particulièrement sur les pronoms personnels, il ne faut toutefois pas oublier les pronoms d'autres natures, bien représentés dans le corpus soumis aux différents contributeurs à ce volume, tels que les pronoms relatifs, les pronoms interrogatifs, les pronoms indéfinis, les pronoms quantifieurs ou encore les pronoms possessifs et les pronoms démonstratifs. Sans doute pouvons-nous considérer, à la suite de Cornish (1999 : 259), que les pronoms personnels sont les moins marqués, ce qui pourrait expliquer leur fréquence en relation anaphorique et la nécessité d'une contrainte cognitive de saillance pour leur utilisation. Le pronom personnel IT quant à lui, est le moins marqué de tous puisqu'il est de genre neutre et de nombre singulier, caractéristiques qui contribuent sans aucun doute à ses emplois impersonnels, et lui permettent, par extension, d'instancier les places laissées vides (de sujet ou d'objet) dans des structures telles que les clivées et les extrapositions. Inversement, les autres types de pronoms contribuent en discours à ajouter de l'ostention (les démonstratifs), des marques d'indétermination (les interrogatifs, les indéfinis) ou de précisions (les quantifieurs, les possessifs), ou encore des marques de relativisation et de thématisation dans une structuration complexe (les relatifs, dont le pronom relatif $\varnothing$ en anglais), ... Le lien entre les pronoms interrogatifs et les pronoms relatifs a été particulièrement exploré par les approches fondées sur l'énonciation. Les deux groupes de pronoms partagent certaines formes en WH- en anglais et en QU- en français (who, which, whom, where, whose, "qui », "que ») : le lien émane du sémantisme de WH- et de QU-, marqueurs d'un manque référentiel, rapidement comblé par l'antécédent dans le cas des pronoms relatifs mais ouvrant un parcours des référents possibles dans le cas des pronoms interrogatifs. 
D'autres aspects des pronoms restent à mettre en évidence, notamment par l'enrichissement mutuel d'approches théoriques différentes, ce qui fait l'objet de la partie suivante.

\subsection{Quel dialogue, quels apports sur les pronoms, dans ce volume?}

Les contributions réunies dans ce volume reflètent un questionnement essentiel portant sur la délimitation de la catégorie des pronoms en anglais. Contrairement à l'anaphore qui constitue un processus, les pronoms sont des marqueurs, et à ce titre, il devrait être possible de les identifier et d'en faire une liste fermée. Mais sur quels critères? Un critère sémantique est que les pronoms permettent "d'assurer une certaine économie dans le discours, en évitant les répétitions et en fondant leur interprétation sur le contexte et la situation » (texte de cadrage qui a accompagné le corpus commun d'où sont extraites les données analysées dans ce volume). Les données diachroniques permettent par ailleurs d'isoler différentes sous-catégories de pronoms, ainsi que les présente C. Delesse dans ce volume: les pronoms personnels, les possessifs, les réfléchis, les démonstratifs, les interrogatifs, les relatifs et les indéfinis.

Toutefois, cela suffit-il pour constituer une liste fermée de pronoms? Les critères d'appartenance à la catégorie (voire la sous-catégorie) des pronoms ne sont pas faciles à établir, ainsi que le montre la contribution de J. Puckica (ce volume). En effet, si les marqueurs pressentis remplissent bien les critères de distribution des noms, ils ont aussi une grande hétérogénéité aux plans morphologique (pronoms en WH-, pronoms réfléchis en -SELF, etc.) et sémantique, puisque certains sont typiquement anaphoriques, d'autres typiquement déictiques, et d'autres encore ne peuvent être ni l'un, ni l'autre : pour ce dernier cas, J. Puckica cite notamment « les indéfinis composés en some-, any-, no- +-body, -one, -thing, le pronom générique one, les interrogatifs what? et who?, ou encore le "relatif indéfini » whoever». J. Puckica propose alors de retenir l'absence de détermination comme critère commun aux divers pronoms et de définir ces derniers comme "une sous-classe fermée de noms qui ne prennent pas de déterminant, dont les membres centraux fonctionnent typiquement de façon anaphorique ou déictique ». J. Puckica discute par ailleurs des liens étroits qui existent entre les pronoms et les déterminants de la tradition et du bien-fondé d'une distinction entre ces deux classes. Il suggère que cette distinction est utile pour les articles et les pronoms personnels mais plus discutable pour d'autres " couples " déterminantpronom (this det $_{\text {vs }}$ this $s_{\text {prn }}$, some $e_{\text {det }}$ vs some $e_{\text {prn }}$, etc.).

Un autre point à l'étude dans les différentes contributions est le rôle joué par les pronoms, outre celui d'économie dans le discours déjà mentionné. Quelles convergences et quelles divergences relève-t-on selon les approches?

60 Pour la perspective cognitive, présentée par L. Gardelle, les pronoms personnels tiennent comme souvent une place prépondérante au sein des pronoms, suivis par les démonstratifs. On retiendra cependant qu'ils ne sont pas vus comme les " parents pauvres » des SN en déterminant + nom. Dans les théories cognitives de la référence, ils occupent ainsi l'extrémité des échelles proposées, et ont un contenu positif : référent saillant, continuité d'attention. En Grammaire Cognitive, par ailleurs, ils occupent également une large place dans les analyses. Ce modèle cherche à proposer une définition des pronoms (personnels et démonstratifs principalement), au sein de l'étude plus large des stratégies d'accès à la référence. Ils se caractérisent par leur 
fonction référentielle ; cela vaut même pour le IT des extraposées, par exemple, qui n'est donc pas juste un « explétif ». Plus spécifiquement, les pronoms allient un ancrage situationnel, comme les déterminants, et une spécification hautement schématique humain pour HE ou SHE, par exemple.

61 La question du caractère universellement référentiel ou non des pronoms personnels apparaît également en grammaire générative. A. Jugnet, qui se concentre sur les pronoms en usage non anaphorique, montre que les analyses de IT et THERE «explétifs» divergent sur ce point: non référentiels pour certains parce qu'ils ne peuvent pas être remplacés par un SN (on voit la primauté au SN sur les pronoms ici), ils sont plutôt "quasi-arguments" pour d'autres. Les études génératives ont par ailleurs pour spécificité de mettre en avant le prisme de la syntaxe: contraintes syntaxiques sur les verbes associables à THERE (inaccusatifs), approche par transformations ou par cas d'orphelinage pour certains emplois de pronoms en WH-.

De façon sous-jacente dans les autres contributions, en cohérence avec les développements ci-dessus, on relève finalement que les pronoms, parce que leur contenu sémantique est allégé et schématisé, ont pour rôle de renvoyer à des entités dans le cadre du discours qu'ils contribuent à structurer et dont ils sont dépendants.

63 L'analyse des pronoms se prête par ailleurs à effectuer des regroupements entre les sous-catégories et à les traiter en paradigmes sur la base des fonctionnements observés. Toutefois, il est frappant de constater que ces regroupements ne sont pas nécessairement les mêmes d'une approche à l'autre, et qu'ils s'effectuent autant sur une base de points communs que sur une base d'oppositions :

- il est possible de regrouper d'une part les pronoms qui véhiculent directement un référent extralinguistique, comme le font les pronoms démonstratifs, des pronoms tels que les relatifs qui n'évoquent rien directement

65 - THERE et IT explétifs sont regroupés dans la présentation en grammaire générative (A. Jugnet, ce volume) sur la base de leur rôle syntaxique, puis distingués sur un critère référentiel, IT étant davantage porteur de référentialité que THERE; dans la perspective cognitive, les deux termes sont regroupés sur la base de leur charge sémantique, de leur caractère référentiel malgré les apparences; Langacker rejette également toute forme de transformation ou réagencement des énoncés; c'est là une grande différence avec des approches telles que la grammaire générative ou même peut-être la syntaxe génétique.

66 - pronoms personnels et pronoms démonstratifs se distinguent dans la perspective cognitive par leur mode de référence, l'un sélectionnant le référent sur la base de la personne, l'autre sur une opération d'ostension (L. Gardelle, ce volume)

67 - pronoms interrogatifs et pronoms relatifs partagent le morphème WH-, ce qui leur vaut d'être potentiellement liés par une valeur sémantique pour Langacker (perspective cognitive), une hypothèse soutenue par Cotte (1996) qui attribue au WHrelatif la capacité d'arrêter le parcours, signalé par WH-, sur le référent de l'antécédent.

Un autre point traité par les contributions est celui de l'accès au référent que procurent les pronoms. L'échelle d'accessibilité d'Ariel, bien connue, est reprise dans les contributions de L. Gardelle et de S. Wilhelm. Elle considère surtout les pronoms personnels et démonstratifs; ils correspondent à des degrés d'accessibilité différents. Cette échelle peut être affinée par la «Hiérarchie du Donné » de Gundel et al., cités par L. Gardelle, dans laquelle chaque type de forme a un «niveau de saillance 
cognitive préféré». L'accessibilité est clairement en rapport avec l'atténuation phonétique, et l'étude phonologique menée par $\mathrm{S}$. Wilhelm illustre bien les différents degrés sur lesquels il est possible de situer les pronoms sur la base de leur lien entre sens et accentuation; ainsi, les pronoms à faible charge sémantique par manque de référenciation directe dans l'extralinguistique (comme les relatifs) sont porteurs de peu d'accentuation, ceux qui sont plus chargés sémantiquement et ont "une référence immédiate dans l'extralinguistique » (S. Wilhelm). Ainsi, les pronoms démonstratifs sont accentués, tandis que les relatifs fusionnés, qui se situent dans un intermédiaire sémantique, n'ont pas de forme faible.

Quant aux données d'acquisition (C. Rossi \& L. Vincent-Durroux), elles montrent notamment que les pronoms présentant des formes accentuées sont acquis en premier, notamment les démonstratifs, chez l'enfant à développement typique, alors que l'enfant à développement atypique est confronté à des limitations cognitives potentiellement liées aux opérations complexes que requiert la pronominalisation.

\section{Anaphore et pronoms en dialogue}

70 Force est de constater au terme de ce parcours qu'étudier l'anaphore ne peut guère se faire sans pronoms, et inversement. Il s'agit bien sûr d'une convergence partielle. Comme l'a rappelé Pierre Cotte durant un échange, l'anaphore est une fonction, par définition transversale par rapport aux classes de mots, et concerne surtout des constituants; les pronoms, eux, ont pour vocation d'être une classe de mots. Mais anaphore et pronoms ont vocation à co-exister dans une théorie plus large de la référence: si pour certaines approches, quelques usages pronominaux ne sont pas référentiels (quantifieurs, explétifs), d'autres cherchent au contraire à "sauver » la fonction référentielle même pour ces usages, pour en faire une caractéristique fondamentale de la classe - on l'a vu notamment avec la Grammaire Cognitive. Ce lien central à la référence explique également la place particulière de la $3^{\mathrm{e}}$ personne : elle seule mobilise l'anaphore (la $1^{\text {ère }}$ et la $2^{\mathrm{e}}$ sont déictiques), et elle seule présente des concurrences paradigmatiques, au sein de la classe des pronoms (ex. pronoms personnels vs. démonstratifs) et au-delà (ex. pronoms vs. SN développés). Plus largement, la notion de référence, qu'elle soit située dans le cadre large de l'anaphore ou le cadre plus restreint et applicatif de l'emploi des pronoms, a entraîné un questionnement autour de l'antécédent. L'apport des approches cognitives a permis de concevoir l'antécédent autrement que dans une relation syntaxique (Cornish 1999), une conception confirmée par les contributions de ce volume. De fait, même si un antécédent semble repérable dans la production ou le contexte, il est désormais admis que le contour référentiel de l'antécédent est à situer au-delà de la syntaxe et des mots. Comprendre ainsi la notion d'antécédent libère considérablement du biais d'une relation terme à terme, et ouvre des pistes pour analyser l'évolution de l'information référentielle, avec le cas emblématique des référents évolutifs, illustrés par les recettes de cuisine (Goux \& Rossi-Gensane 2019).

71 La spécificité du lien entre anaphore et pronoms réside également dans le caractère éminemment syntaxique qu'ils ont tous les deux : l'anaphore ne peut s'étudier qu'en syntaxe (proposition ou discours plus large); le pronom a pour vocation à être un constituant, donc à instancier une fonction syntaxique (d'où les variations en cas pour certains) ; la question de la position est également importante pour les interrogatifs, 
exclamatifs ou relatifs. Or la tradition grammaticale a projeté de lourdes attentes quant à la syntaxe idéale: parler de "substitut abréviatif » d'un SN pour un pronom anaphorique, c'est attendre le SN comme constituant roi en syntaxe; parler de transformations ou de réagencement pour les "explétifs", c'est considérer un seul ordre syntaxique roi. Ce qu'ont montré différentes modélisations, c'est le progrès effectué grâce à des approches plus récentes pour dépasser cette tradition, non pas pour lui ôter toute légitimité, mais pour ne pas être aveuglé. Ainsi, le pronom personnel a été réhabilité ; l'idée de mots dénués de sens (explétifs) est débattue; le réagencement ne fait plus l'unanimité ; ou encore, $\mathrm{S}$. Wilhelm propose de considérer les marqueurs supra-segmentaux comme de véritables marqueurs linguistiques. Ces progrès existent bien sûr aussi dans d'autres domaines des études syntaxiques : il n'est ainsi plus unanimement admis que le passif soit un réagencement de l'actif, ou encore qu'un segment " elliptique » soit obtenu par effacement d'une structure plus complète. Quitter un instant la tradition, c'est aussi peut-être mieux y revenir - pour partie. Grâce au dialogue instauré entre les différentes approches présentes dans ce volume, et au regard croisé sur l'anaphore et les pronoms, on comprend mieux pourquoi le linguiste est toujours ramené, même contre son gré, aux pronoms personnels de $3^{\mathrm{e}}$ personne et démonstratifs. Définis, porteurs d'un sens schématique (genre ou distance/proximité, nombre) suffisamment peu précis pour donner accès à tous types de référents (vs. everything ou mine, par exemple), mais permettant des instructions claires pour accéder à eux en contexte (sens schématique, statut cognitif du référent, cas ou fonction syntaxique), ils sont les outils idéaux d'une grammaire de la référence, et plus particulièrement, pour la troisième personne, de l'anaphore.

L'étude d'autres catégories serait bien sûr pertinente pour éclairer le processus anaphorique, plus particulièrement lorsqu'elles comportent des items fondés sur le marqueur TH- : certains déterminants (THE), certains adverbes (THERE, THEN mais aussi SO, cité ci-dessus dans la locution DO SO), certaines conjonctions (THOUGH) mettent également en jeu l'anaphore, ainsi que la préconstruction, dont le lien mériterait d'être davantage exploré.

74 Au terme de l'étude, il semble que les questionnements initiaux se soient déplacés et soient devenus plus complexes en intégrant des paramètres cognitifs potentiellement coûteux, révélés en creux dans le discours des locuteurs présentant des difficultés, qu'il s'agisse de troubles du langage ou d'accès à la parole (C. Rossi \& L. Vincent-Durroux, ce volume) ou de dégradations cognitives comme la maladie d'Alzheimer (Fossard 2012) ou les syndromes aphasiques. Ainsi, si l'anaphore et les pronoms peuvent contribuer à la production d'un discours moins redondant, plus économique aux plans pragmatiques et référentiels, cela suppose des compétences cognitives dont l'exploration reste également à poursuivre.

BIBLIOGRAPHIE

Adamczewski, H. (1982). Grammaire linguistique de l'anglais. Paris : Armand Colin. 
Allan, K. (2009). Concise Encyclopedia of Semantics. Oxford: Elsevier.

Ariel, M. (1990). Accessing Noun-Phrase Antecedent. London: Routledge.

Auwera, J. van der (1985). Relative that: A centennial dispute. Journal of Linguistics, 21.1, 149-179.

Benveniste, E. (1966). La nature des pronoms. Problèmes de linguistique générale, 1. Chapitre XX.

Paris : Editions Gallimard.

Bhat, D.N.S. (2004). Pronouns. Oxford: Oxford University Press.

Blanche-Benveniste C. (2008). Les unités de langue écrite et de langue parlée. In Bilger, M. (dir.), Données orales - Les enjeux de la transcription. Cahiers de l'Université de Perpignan, 37, 192-217. Perpignan : Presses Universitaires de Perpignan.

Blanche-Benveniste C., Deulofeu J., Stefanini J. \& van den Eyden, K. (1987). Pronom et syntaxe : l'approche pronominale et son application au français. Paris : SELAF (Sociolinguistique, descriptions des langues et interactions sociales et culturelles).

Buyssens, E. (1967). La communication et l'articulation linguistique. Paris : Presses Universitaires de France.

Chastain, C. (1975). Reference and Context. In Gunderson, K. (ed.), Language Mind and Knowledge, 194-269. Minneapolis: University of Minnesota Press.

Corblin, F. (1995). Les formes de reprise dans le discours. Anaphores et chaînes de référence. Rennes : Presses Universitaires de Rennes.

Cornish, F. (1999). Anaphora, Discourse and Understanding: evidence from English and French. Oxford: Clarendon.

Cotte, P. (1996). L'explication grammaticale de textes anglais. Paris : Presses Universitaires de France.

Creissels, D. (1993). Les notions primitives de la syntaxe. Réflexions sur l'articulation logique entre les notions sur lesquelles se fondent les descriptions grammaticales. In Maillard, M. (dir.), Vers une rénovation de la grammaire et de sa terminologie. Lidil 8, 51-83. Grenoble : Presses Universitaires de Grenoble.

Culicover, P. W. (2009). Natural Language Syntax. Oxford: Oxford University Press.

Culicover, P. W. \& Jackendoff R. (2005). Simpler Syntax. Oxford: Oxford University Press.

David, C., Poussard, C. \& Vincent-Durroux, L. (2019). Who is who ? Gestion de l'anaphore en interaction exolingue : le cas de francophones apprenant l'anglais. In Gardelle L., Rossi, C. \& Vincent-Durroux L. (dir.), Cahiers de praxématique 72, La gestion de l'anaphore en discours. http:// journals.openedition.org/praxematique/5493

Debras, C. \& Beaupoil-Hourdel P. (2019). Gestualité et construction des chaînes de référence dans un corpus d'interactions tandem. In Gardelle L., Rossi, C. \& Vincent-Durroux L. (dir.), Cahiers de praxématique 72, La gestion de l'anaphore en discours. http://journals.openedition.org/ praxematique/5576

Ehlich, K. (1982). Anaphora and deixis: Same, similar, or different?. In Jarvella, R. J., Klein, W. (eds.), Speech, place and action. Studies in deixis and related topics, 315-338. Chichester: Wiley.

Flambard, G. (2017). A compositional analysis of VP anaphors. Anglophonia 23. https:// journals.openedition.org/anglophonia/1192

Fossard, M. (2012). Aspects cognitifs de l'anaphore pronominale, II. Editions universitaires européennes. 
Gardelle, L. (2006). Le genre en anglais moderne (XVIe siècle à nos jours) : le système des pronoms. Thèse de doctorat. Université Paris-Sorbonne (Paris 4).

Gardelle, L. (2014). La catégorisation en langue et en discours. Vol. 1 : Synthèse de la recherche. Dossier d'Habilitation à Diriger les Recherches, Université Paris IV [manuscrit].

Gardelle, L. \& Lacassain-Lagoin C. (2012). Analyse linguistique de l'anglais : méthodologie et pratique. Rennes : Presses Universitaires de Rennes.

Gardelle, L., Rossi C. \& Vincent-Durroux L. (2019). La gestion de l'anaphore en discours : complexités et enjeux. In Gardelle L., Rossi, C. \& Vincent-Durroux L. (dir.). Cahiers de praxématique 72, La gestion de l'anaphore en discours.

https://journals.openedition.org/praxematique/5319

Goux, M. \& Rossi-Gensane, N. (2019). Référents évolutifs, anaphores et constructions détachées : étude diachronique de recettes de cuisine. In Gardelle L., Rossi, C. \& Vincent-Durroux L. (dir.), Cahiers de praxématique 72, La gestion de l'anaphore en discours.

http://journals.openedition.org/praxematique/5424

Gundel, J.K., Hedberg, N. \& Zacharski, R. (1993). Cognitive status and the form of referring expressions in discourse. Language 69, 274-307.

Haegeman, L. \& Guéron, J. (1999). English Grammar: A Generative Perspective. Blackwell Textbooks in Linguistics. Oxford: Blackwell.

Houser, M.J. (2010). The Syntax and Semantics of Do So Anaphora. Ph.D. dissertation, University of California, Berkeley.

Irmer, M. (2011). Bridging Inferences. Constraining and resolving under-specification in discourse interpretation. Berlin: de Gruyter.

Khalifa, J.-C. (1999). La syntaxe anglaise aux concours CAPES / Agrégation. Théorie et pratique de l'énoncé complexe. Paris : Armand Colin.

Kleiber, G. (2001). L'anaphore associative. Paris : Presses Universitaires de France.

Langacker, R.W. (1969). On pronominalization and the chain of command. In Shane, S.A. \& Reibel, D.A. (eds.), Modem Studies in English: Readings in Transformational Grammar, 160-186. Englewood Cliffs, NJ: Prentice Hall.

Lapaire, J.-R. \& Rotgé W. (1991). Linguistique et grammaire de l'anglais. Toulouse : Presses Universitaires du Mirail.

Mignot, E. (2012). The conceptualization of natural gender in English, Anglophonia / Sigma 32, 3961.

Mignot, E. (2016). Linguistique anglaise. Paris : Armand Colin.

Miller, P. (2011). The choice between verbal anaphors in discourse. In Hendricks, I., Lalitha Devi, S., Branco, A. \& Mitkov, R. (eds.), Anaphora Processing and Applications. 8th Discourse Anaphora and Anaphor Resolution Colloquium, DAARC2011. Berlin: Springer, 82-95. https://sites.google.com/site/ philipmillerlinguistics/publications/articles

Miller, P. (2013). Usage preferences: The case of the English verbal anaphor do so. In Müller, S. (ed.) Proceedings of the 20th International Conference on Head-Driven Phrase Structure Grammar, 121139. CSLI publications. http://csli-publications.stanford.edu/HPSG/2013

Quine, W. (1964). From a Logical Point of View. Harvard, MA: Harvard University Press. 
Reichler-Béguelin, M.-J. (1993). Anaphores associatives non lexicales: incomplétude macrosyntaxique? In Karolak, S. \& Muryn, T. (dir.), Complétude et incomplétude dans les langues romanes et slaves. Actes du 6e Colloque international de Linguistique romane et slave (Cracovie, 29 sept. - 3 oct. 1991), 327-379. Cracovie : Wyd. Naukowe WSP. http://www2.unine.ch/files/content/sites/ linguistique.francaise/files/shared/documents/Anaphores_associatives_non_lexicales.PDF

Reinhart, T. (1976). The Syntactic Domain of Anaphora. Ph.D. dissertation, MIT.

Reinhart, T. (1981). Definite NP-anaphora and c-command domains. Linguistic Inquiry 12, 605-635.

Salzar-Orvig, A. \& Grossen, M. (2010). La co-construction : une facette dialogale du dialogisme? Colloque international Dialogisme : langue, discours. Montpellier. www.praxiling.fr/IMG/ pdf_SalazarGrossen1.pdf

Schnedecker, C. (1997). Nom propre et chaînes de référence. Paris : Klincksieck.

Schnedecker, C. (2014). Chaînes de référence et variations selon le genre. Langages 195(3), 23-42.

Schnedecker, C. \& Landragin F. (2014). Les chaînes de référence : présentation. Langages 195(3), 322.

Schwartz-Friesel, M. (2007). Indirect anaphora in text: a cognitive account. In Schwartz-Friesel, M. et al. (eds.), Anaphors in Text: cognitive, formal and applied approaches to anaphoric reference, 3-20. Amsterdam: John Benjamins.

Sportiche, D., Koopman, H. \& Stabler, E. (2014). An Introduction to Syntactic Analysis and Theory. Chichester: Wiley Blackwell.

Stefanini, J. (1976). Jules César Scaliger et son De Causis Linguae Latinae. In Parret, H. (ed.), History of Linguistic Thought and Contemporary Linguistics, 317-330. Berlin: Walter de Gruyter.

Zribi-Hertz, A. (1996). L'anaphore et les pronoms. Une introduction à la syntaxe générative. Lille : Presses Universitaires du Septentrion.

\section{NOTES}

1. Les coordinatrices $d u$ volume remercient les membres du comité scientifique pour leur relecture et leurs conseils : Catherine Delesse, Olivier Glain, Sophie Herment, Anne Jugnet, Laure Lansari, Anne Mathieu, Philip Miller, Aliyah Morgenstern et Jérôme Puckica.

2. C-commande (traduction de Zribi-Hertz 1996 : 56, par exemple) : Le nœud A c-commande le nœud B si et seulement si (i) A ne domine pas B et B ne domine pas A et (ii) le premier nœud à ramifications qui domine A domine aussi B. Liage : A lie B si et seulement si (i) A c-commande B et (ii) A et B sont co-indexés.

3. Très occasionnellement, d'autres cas d'anaphore sont mentionnés : ainsi, chez Culicover \& Jackendoff (2005 : 283), X happen anaphora (ex. it / that / the same thing / something similar / ... happened) et One-anaphora (ex. Leslie read a long book about Lincoln, and Steve read a short one about Stalin).

4. Pour Schwartz-Friesel (2007), l'anaphore associative est un cas d'anaphore indirecte ; mais il est à noter que cette classification ne fait pas l'unanimité. Ainsi Kleiber (1981: 53) suggère que l'anaphore associative ne répond pas au même processus référentiel que les anaphores indirectes (de type ils grégaire ou autres).

5. «-ING pourrait être utilisé pour signaler le caractère anaphorique du segment qu'il domine. Par 'anaphorique' il faut entendre acquis pour l'énonciateur car déjà repéré, déjà construit mentalement, déjà exprimé ou logiquement prévisible ('donné / acquis d'avance'). En ce sens, 
-ING relèverait d'un processus mémoriel de 'retour de l'esprit sur son propre passé' (l'expression est empruntée à G. Guillaume)» (Lapaire \& Rotgé 1991: 417 - gras présent dans l'original, comme dans toutes les citations de ce chapitre).

\section{AUTEURS}

\section{LAURE GARDELLE}

Université Grenoble Alpes - Laboratoire Lidilem (Linguistique et didactique des langues étrangères et maternelles)

\section{LAURENCE VINCENT-DURROUX}

Université Grenoble Alpes - Laboratoire Lidilem (Linguistique et didactique des langues étrangères et maternelles) 